\title{
On the Reduction Formula of Feinberg and Pais
}

C. G. Bollini, J. J. Giambiagi, and A. González Domínguez

Citation: Journal of Mathematical Physics 6, 165 (1965); doi: 10.1063/1.1704256

View online: https://doi.org/10.1063/1.1704256

View Table of Contents: http://aip.scitation.org/toc/jmp/6/1

Published by the American Institute of Physics

\section{PHYSICS TODAY}

WHITEPAPERS
MANAGER'S GUIDE

Accelerate R\&D with Multiphysics Simulation
READ NOW

PRESENTED BY OCOMSOL 


\title{
On the Reduction Formula of Feinberg and Pais
}

\author{
C. G. Bollini and J. J. Giambiagi \\ Departamento de Física, Facultad de Ciencias Exactas y Naturales, Buenos Aires, Argentina \\ AND \\ A. González Domínguez \\ Departamento de Matemática, Facultad de Ciencias Exactas y Naturales, Buenos Aires, Argentina
}

(Received 14 July 1964)

Feinberg and Pais have considered a "reduction" formula for the Fourier transform of functions which depend only on the hyperbolic distance. They have shown that the formula is valid in particular cases and stated that it should be valid in general.

We show that it is valid for any causal distribution and furthermore that it is actually an extension of the well-known Bochner Theorem on the Fourier transform of radial functions.

\section{INTRODUCTION}

TN their work on weak interactions, Feinberg 1 and Pais" have used the following "reduction" formula ${ }^{2}$ :

$$
\begin{aligned}
I\left(q^{2}\right) & =\int \psi\left(y^{2}\right) e^{i \alpha \cdot y} d^{4} y=F(\psi), \\
I\left(q^{2}\right) & =\frac{4 i \pi^{2}}{q}\left[\int_{c} H_{1}^{(1)}(q y) \psi\left(y^{2}\right) y^{2} d y\right. \\
& \left.\quad-\int_{0}^{\infty} J_{1}(q y) \psi\left(y^{2}\right) y^{2} d y\right],
\end{aligned}
$$

where

$y^{2}=y_{1}^{2}+y_{2}^{2}+y_{3}^{2}-y_{4}^{2}, \quad q^{2}=q_{1}^{2}+q_{2}^{2}+q_{3}^{2}-q_{4}^{2}$.

In all the cases considered by Feinberg and Pais, ${ }^{1}$ it turns out that the contour integral vanishes. Furthermore, they state: "We are convinced, that any alternative treatment of the contour integral leads to physical absurdities." ${ }^{\prime 3}$ This means that, in fact

$$
F\left(\psi\left(y^{2}\right)\right)=-\frac{4 i \pi^{2}}{q} \int_{0}^{\infty} J_{1}(q y) \psi\left(y^{2}\right) y^{2} d y
$$

We would like to point out that the latter formula is actually the causal version of the following wellknown theorem, due to Bochner ${ }^{4}$ (which we are writing for a four-dimensional space):

If

$$
f\left(y_{1} y_{2} y_{3} y_{4}\right) \in L_{1}
$$

depends only on $R=\left(y_{1}^{2}+y_{2}^{2}+y_{3}^{2}+y_{4}^{2}\right)^{\frac{1}{2}}$, then

${ }^{1}$ G. Feinberg and A. Pais, Phys. Rev. 131, 2724 (1963).

2 Reference 1, p. 2735. See also, Y. Pwu and T. T. Wu, Phys. Rev. 133, B 778 (1964).

Reference 1, p. 2738.

4 S. Bochner and K. Chandrasekharan, Fourier Transform (Princeton University Press, Princeton, New Jersey, 1949), p. 69. the function

$$
\phi\left(q_{1} q_{2} q_{3} q_{4}\right)=\int f\left(y_{1} y_{2} y_{3} y_{4}\right) e^{i \cdot \cdot y} d^{4} y
$$

depends only on

$$
\rho=\left(q_{1}^{2}+q_{2}^{2}+q_{3}^{2}+q_{4}^{2}\right)^{\frac{1}{3}} .
$$

Furthermore,

$$
\phi(\rho)=\frac{4 \pi^{2}}{\rho} \int_{0}^{\infty} f(R) J_{1}(\rho R) R^{2} d R .
$$

In the applications to physics of form (3), $\psi\left(y^{2}\right)$ is not, in general, an ordinary function but a distribution. What is actually needed for our purpose is formula (4) for the case in which $f$ is a tempered distribution." This extension has been done by one of us.

\section{CAUSAL DISTRIBUTIONS}

We shall first define the "causal" distributions." Starting with a radial distribution, ${ }^{6}$ which will be simply written as $\psi\left(y_{1}^{2}+y_{2}^{2}+y_{3}^{2}+y_{4}^{2}\right)$, we introduce a positive parameter $a$ by means of the following definition $\left(\varphi\right.$ is a test function $\left.\in S^{5}\right)$ :

$$
\begin{array}{r}
\left(\psi_{a}, \varphi\right)=\left(\Psi\left(y_{1}^{2}+y_{2}^{2}+y_{3}^{2}+a y_{4}^{2}\right), \varphi\left(y_{1}, y_{2}, y_{3}, y_{4}\right)\right) \\
\equiv \frac{1}{a^{\frac{1}{2}}}\left(\Psi\left(R^{2}\right), \varphi\left(y_{1}, y_{2}, y_{3}, \frac{y_{4}}{a^{\frac{1}{3}}}\right)\right) .
\end{array}
$$

As usual, $\Psi_{a}$ will be said to be analytic in $a$ if for any test function $\varphi$, the ordinary function $\left(\psi_{a}, \varphi\right)$ is analytic in $a .^{8}$ When $\Psi_{a}$ can be analytically continued into the whole of the upper half-plane of $a$, we define

${ }^{5} \mathrm{~L}$. Schwartz, Theorie des distributions (Hermann \& Cie., Paris, 1951), Vol. 2.

- A. González Dominguez (to be published).

7 We follow the steps discussed by I. M. Guelfand and G. E. Shilov, Les distributions (Dunod Cie., Paris, 1962), in particular Chap. III, 2, p. 264.

8 Reference 7, p. 148. 
\& causal distribution by the formula

$\Psi\left(y^{2}+i 0\right)=\lim _{a \rightarrow-1+i 0} \psi\left(y_{1}^{2}+y_{2}^{2}+y_{3}^{2}+a y_{4}^{2}\right)$.

An "anticausal" distribution can be defined as the complex conjugate of a causal one.

We now show that it is possible to compute the Fourier transform of a causal distribution by means of Formula (3). In fact,

$$
\begin{aligned}
(I, \varphi) \equiv & (F(\psi), \varphi)=\left(\psi, F^{-1}(\varphi)\right) \\
& =\lim _{a \rightarrow-1+i 0}\left(\psi_{a}, F^{-1}(\varphi)\right)=\lim _{a \rightarrow-1+i 0}\left(F\left(\psi_{a}\right), \varphi\right)
\end{aligned}
$$

i.e.,

$$
I=\lim _{a \rightarrow-1+i 0} F\left(\psi_{a}\right)=\lim _{a \rightarrow-1+i 0} I_{a}
$$

where

$I_{a}\left(q_{1}, q_{2}, q_{3}, q_{4}\right)=\int \psi\left(y_{1}^{2}+y_{2}^{2}+y_{3}^{2}+a y_{4}^{2}\right) e^{i a \cdot y} d^{4} y$.

For positive $a$ we have

$$
\begin{aligned}
& I\left(q_{1}, q_{2}, q_{3}, \frac{q_{4}}{a^{\frac{1}{3}}}\right) \\
& \quad=\frac{1}{a^{I}} \int \psi\left({y_{1}^{\prime 2}}^{2}+{y_{2}^{\prime 2}}^{\prime 2}+{y_{3}^{\prime 2}}^{\prime 2}+y_{4}^{\prime 2}\right) e^{i_{a^{\prime} y^{\prime}}} d^{4} y,
\end{aligned}
$$

where the fourth component of $q_{\alpha}^{\prime}$ is $q_{4} / a^{\sharp}$.

Now, using Bochner theorem [Formula (4)], we obtain

$$
\begin{aligned}
I\left(\rho^{2}\right)=I\left(q_{1}^{2}+\right. & \left.q_{2}^{2}+q_{3}^{2}+\frac{1}{a} q_{4}^{2}\right) \\
& =\frac{4 \pi^{2}}{a^{\frac{1}{2}} \rho} \int_{0}^{\infty} \psi\left(R^{2}\right) J_{1}(\rho R) R^{2} d R .
\end{aligned}
$$

Formula (6) is valid for positive $a$. By making the

\footnotetext{
${ }^{\circ}$ See Ref. 5, p. 106.
}

analytic continuation in the upper half-plane of the parameter $a$ and taking the limit $a \rightarrow-1+i 0$, we obtain

$$
\begin{aligned}
I\left(q_{1}^{2}+q_{2}^{2}+q_{3}^{2}\right. & \left.+q_{4}^{2}\right) \equiv I\left(q^{2}\right) \\
& =\frac{4 \pi^{2}}{i q} \int_{0}^{\infty} \psi\left(R^{2}\right) J_{1}(q R) R^{2} d R,
\end{aligned}
$$

which coincides with formula (3). [It is understood that $I\left(q^{2}\right)$ means $I\left(q^{2}-i 0\right)$; see Formula (5).]

Formula (8) is valid for causal distributions. For anticausal ones the result is

$$
I\left(q^{2}\right)=-\frac{4 \pi^{2}}{i q} \int_{0}^{\infty} \psi\left(R^{2}\right) J_{1}(q R) R^{2} d R,
$$

where it is understood that $q^{2}$ means $q^{2}+i 0$.

\section{EXAMPLE}

As an example of application we consider now the causal distribution

$$
\psi\left(y^{2}\right)=\left(y^{2}+i 0\right)^{\lambda},
$$

where $\lambda$ is any complex number. A direct application of Formula (8) gives $^{10}$ :

$I\left(q^{2}\right)=\frac{4 \pi^{2}}{i q} \int_{0}^{\infty} J_{1}(q y) y^{2 \lambda+2} d y=\frac{4 \pi^{2}}{i} \frac{2^{2 \lambda+2} \Gamma(\lambda+2)}{\left(q^{2}\right)^{\lambda+2} \Gamma(-\lambda)}$, which is the correct result. ${ }^{11}$

It should be noted from (10) that, near the origin, $\psi\left(y^{2}\right)$ has a singularity worse than $y^{-2}$ when $\lambda<-1$. Nevertheless, Formula (8) is still valid, although the contour integral considered by Feinberg and Pais is not meaningful in this case.

${ }^{10} \mathrm{~W}$. Groebner and N. Hofreiter, Integraltafel (Bestimmte Integrale) (Springer-Verlag, Berlin, 1958), p. 196. Also Formula (1), p. 198, analytically extended in $k$.

11 Reference 7, p. 278. 